\title{
Agentes tutores para la enseñanza
}

\author{
Diego-Fernando Marcillo-Pinzón ${ }^{1}$
}

Fecha de recepción: 1 de abril de 2020

Fecha de aprobación: 19 de mayo de 2020

\section{Resumen}

El artículo inicia analizando los estudios realizados sobre los agentes tutores para la enseñanza, llevándose a cabo una búsqueda bibliográfica amplia y exhaustiva de artículos relacionados, todo con el fin de conseguir un mayor conocimiento sobre la aplicabilidad del tema y por medio del cual se pueda utilizar en aplicaciones futuras. Los métodos de investigación utilizados fueron el descriptivo y exploratorio, los cuales se ajustaron, logrando recopilar los datos necesarios. La investigación permitió demostrar como la aplicación de la tutoría en la educación se presenta como un agente de superación para diferentes problemas evidenciados en los procesos de aprendizaje. Una efectiva aplicación elimina las desigualdades de estudiantes en el contexto académico, lo cual es un aporte importante en búsqueda de mejorar la calidad de la educación. Los buenos resultados obtenidos en los centros educativos replantean el concepto del aprendizaje en metodologías usadas actualmente, enfocando la tutoría como un derecho del estudiante en su vida académica. En la aplicación debe existir un apoyo de todos los roles involucrados en el aprendizaje (profesores e instituciones educativas), lo cual garantice un éxito en su aplicación.

Palabras clave: enseñanza; STI; tutor virtual; tutoría.

\footnotetext{
${ }^{1}$ Universidad de la Amazonia (Florencia-Caquetá, Colombia). d.marcillo@udla.edu.co.
} 


\section{Tutors Agents for Teaching}

\section{Abstract}

The article begins by analyzing the studies carried out on tutors for teaching, carrying out a wide and exhaustive bibliographic search for related articles, all with the aim of obtaining a better knowledge of the applicability of the topic and through which it can be used in future applications. The research methods used were descriptive and exploratory, which were adjusted, managing to collect the necessary data. The research allowed demonstrating how the application of tutoring in education is presented as an agent for overcoming different problems evident in the learning processes. An effective application eliminates the inequalities of students in the academic context, which is an important contribution in search of improving the quality of education. The good results obtained in educational centers rethink the concept of learning in currently used methodologies, focusing on tutoring as a right of the student in her academic life. In the application there must be support from all the roles involved in learning (teachers and educational institutions), which guarantees success in its application.

Keywords: STI; teaching; tutorships; virtual tutor.

Para citar este artículo:

Marcillo-Pinzón, D.-F. (2020). Agentes tutores para la enseñanza. Pensamiento y Acción, $28,61-82$.

Esta obra está bajo licencia internacional Creative Commons Reconocimiento 4.0 


\section{Introducción}

En el siguiente trabajo se realizó una búsqueda exploratoria de diferentes artículos relacionados con los agentes en la enseñanza, los cuales se muestran como soluciones para los distintos problemas encontrados en la educación, tiendo en cuenta lo anterior, el trabajo tiene como objetivo general, el análisis de los estudios realizados sobre los agentes tutores para la enseñanza. Teniendo en cuenta la poca información encontrada sobre el tema, es desconocido el impacto de la aplicación de esta metodología. Existe la necesidad de aplicar la tutoría en la educación, para dar solución a los problemas evidenciados en el aprendizaje, encarando las desigualdades de los alumnos en el contexto académico (Narro \& Arredondo, 2013).

El uso de las TIC en el aula proporciona una mejor gestión en el proceso de enseñanza-aprendizaje, implementando nuevos entornos en búsqueda de solucionar las necesidades de los estudiantes, es así como se convierte en un recurso motivador e innovador en comparación con metodologías antiguas y poco eficientes (Garrido \& García, 2016). La tutoría inclusiva virtual en educación a distancia beneficia el desarrollo cognitivo en los estudiantes involucrados, para su aplicación es importante el rol del tutor virtual, el cual sea un agente facilitador para la construcción de ambientes colaborativos, dentro del grupo de tutoría debe existir un motivador para crear buenas sensaciones y un ambiente propicio en la mejora del aprendizaje. La utilización de una metodología adecuada es relevante en el éxito de la aplicación, donde se pueda generar buenas sensaciones y resultados académicos (Hernández-Sánchez \& Ortega, 2015).

El artículo se clasifica en 3 categorías principales: dos bases y una emergente, cada una se componen de 3 subcategorías. Las categorías bases son las siguientes: 1) agentes tutores, la cual se clasifica en: tutoría educativa en espacios virtuales, tutoría entre iguales y la tutoría en el contexto educativo. 2) La enseñanza, la cual está clasificada en: la gamificación en la educación, las TIC's en la enseñanza y el aprendizaje colaborativo. Finalmente, en la categoría emergente se encuentra: La tutoría en la educación virtual, la cual se clasifica en E-Learning: Tutoría por medio de las tics y Sistemas tutores inteligentes en el ámbito de la educación 


\section{Materiales y métodos}

Se seleccionaron los métodos de investigación exploratorio y descriptivo para llevar a cabo el proceso de búsqueda y construcción de los resultados. El método descriptivo está basado en estudios de ambientes naturales, permitiendo tener una visión general para enfocar las variables relevantes, su plan de trabajo consiste en definir inicialmente las características a describir, expresando cómo se realizarán las observaciones, la recolección de datos y analizar e informar los resultados. El método exploratorio se adecua al basarse en el análisis de un estudio poco abordado, identificando los estudios realizados con el tema, su plan de trabajo consiste en realizar con precisión la formulación del problema, las preguntas de investigación y el planteamiento de una hipótesis.

Los artículos seleccionados se clasificaron en diferentes categorías: bases y emergentes, donde cada una de las categorías se componían de otras subcategorías, como se presenta en la Figura 1, la clasificación por categorías fue un proceso sumamente importante al momento de realizar la búsqueda de los artículos, definiendo con claridad lo deseado a encontrar.

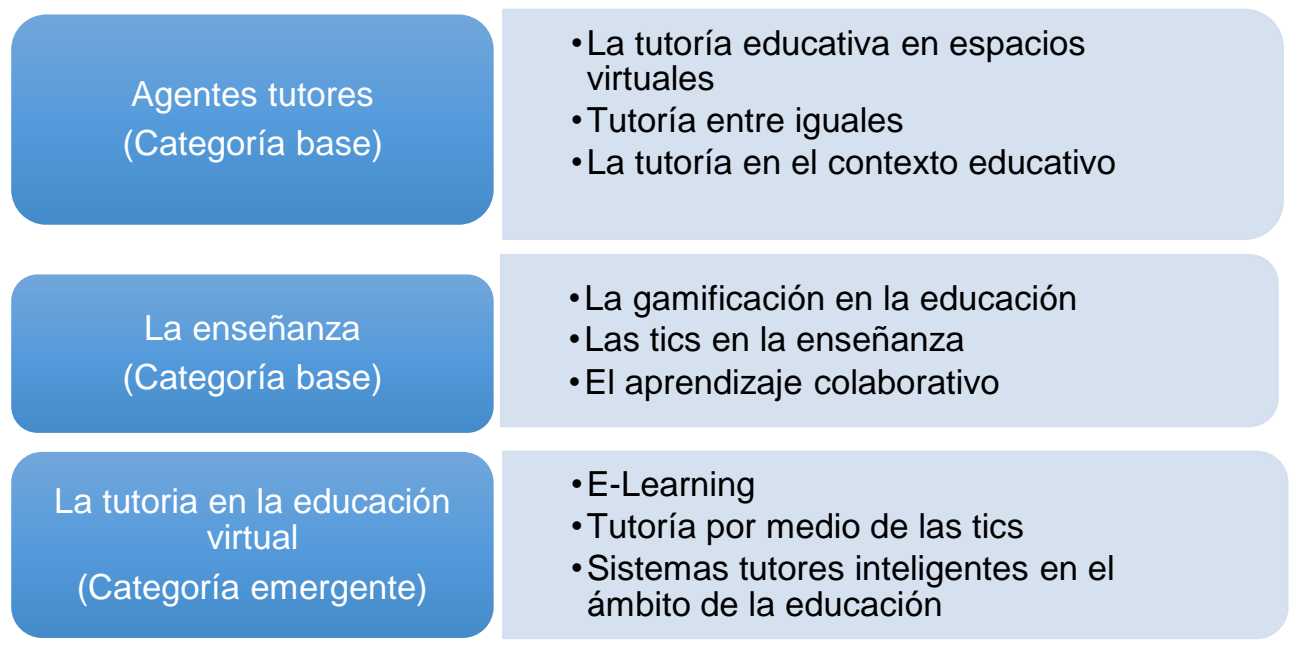

Fig. 1. Clasificación de los temas en categorías.

En la búsqueda de información, se utilizó la herramienta de búsqueda Google Scholar y se encontraron artículos en las diferentes bases de datos especializadas como Scielo, ScienceDirect, Web of Science y IEEE, seleccionando 200 artículos 
relacionados con el tema, al buscar los artículos de forma exploratoria, se logró replantear algunas subcategorías. Para obtener los resultados se establecieron cuatro preguntas de investigación: Pregunta 1 ¿Qué vacíos se presentan en el tema?, pregunta 2 ¿Cómo se desarrolla el tema a lo largo de los años?, pregunta 3 ¿En qué se enfoca la discusión actual? y la pregunta 4 ¿Cuáles son los temas relevantes en estas investigaciones?. Los 51 artículos finales dieron respuesta a las preguntas de investigación, y generaron la construcción de los resultados. En la siguiente tabla se visualiza la información sistematizada de los artículos divididos según el año y pregunta de investigación.

Tabla 1. Clasificación de artículos por año y pregunta de investigación.

\begin{tabular}{|c|c|c|c|c|c|c|c|c|}
\hline Año & Pregunta1 & $\%$ & Pregunta2 & $\%$ & Pregunta4 & $\%$ & Cantidad & Total \% \\
\hline 2013 & 0 & 0 & 0 & 0 & 12 & 23.08 & 12 & 23.08 \\
\hline 2014 & 0 & 0 & 0 & 0 & 5 & 9,61 & 6 & 9,61 \\
\hline 2015 & 1 & 1.92 & 2 & 3.84 & 11 & 21.15 & 14 & 26.91 \\
\hline 2016 & 0 & 0 & 1 & 1.92 & 11 & 21.15 & 12 & 23.07 \\
\hline 2017 & 0 & 0 & 0 & 0 & 9 & 17,30 & 8 & 17,30 \\
\hline Total & & 1.92 & & 5.76 & & 92.31 & 52 & $100 \%$ \\
\hline
\end{tabular}

La mayoría de los artículos analizados fueron publicados en el año 2015 con un total de $26.91 \%$, aunque la diferencia es poca respecto a los años $2013(23.08 \%)$ y $2016(23.07 \%)$. La pregunta de investigación 4 fue la más relacionada con un $92.31 \%$ de los artículos analizados.

\section{Resultados}

Se analizaron los artículos encontrados en la búsqueda bibliográfica, obteniéndose una variación tanto en el tipo de tutoría utilizada, como en el contexto de la aplicación, los resultados obtenidos fueron en su mayoría positivos.

\section{Agentes tutores}

La tutoría se considera fundamental en la educación para enfrentar y superar los diferentes problemas evidenciados en el proceso de enseñanza, no solo se considera una herramienta educativa, sino también un recurso integral (Vallejo Giraldo, 2017). Elimina las desigualdades de alumnos en el contexto académico, lo 
cual ayuda a mejorar la educación ofrecida por las instituciones educativas. La tutoría debe ser considerada como un derecho del estudiante en su vida académica, la apropiación en el uso puede llegar a significar la creación de una experiencia intelectual, contribuyendo a su formación académica y personal (Narro \& Arredondo, 2013).

La tutoría en el contexto universitario es una herramienta generadora de innovación y calidad en el acompañamiento del alumnado, permitiendo una relación académica cercana entre el docente y estudiante (Clares, Cusó, \& Juárez, 2014). Para mejorar la calidad de la aplicación de tutoría debe existir un modelo integral, donde todos los participantes en el proceso sean agentes activos, tanto el profesorado como la institución, reconociendo la importancia del rol docente, fortaleciendo funciones centradas en el estudiante (Rodríguez, 2013)

\section{Tutoría educativa en espacios virtuales}

Las TIC y la tutoría en el aprendizaje universitario reflejan un alto uso de la tutoría virtual, resultado de la valoración de aspectos de fácil acceso y menor tiempo invertido. El uso de la tutoría virtual implica un apoyo desde la función del docente, resaltando la importancia de su inclusión en la educación. La tutoría personal implica mayor tiempo y recursos, aunque la virtual tenga un uso mayor, los estudiantes coinciden en definir la personal como efectiva a sus necesidades (Martínez, Pérez, \& Martínez, 2016). Se evidencia una mayor participación del estudiante cuando enseña, se desarrollan metodología las cuales permitan una fácil construcción de conocimientos, así es como se creó un entorno de tutoría en la web para estudiantes universitarios, donde se implementa la enseñanza por parte de estudiantes por medio de la tutoría entre iguales, con el objetivo de potencializar el aprendizaje y la enseñanza (Park \& Kim, 2015).

La aplicación de la tutoría virtual en educación a distancia refleja la situación del alumno frente a múltiples dificultades para acceder a sus beneficios, falta de tiempo debido a trabajos o por poca motivación hacia el aprendizaje de nuevos conocimientos, esto resulta importante, donde se contribuya a crear un ambiente de orientación y motivación, permitiendo la superación de la deserción (Delgado \& 
Ramos, 2017). Además, se evidencia aspectos de dificultad en su aplicación, principalmente la formación del docente y los difíciles cambios institucionales, debido a largos año de usar metodologías antiguas e ineficientes. La transformación del modelo no tiene éxito sin la voluntad de dichos elementos (Gómez, 2013) (PlazaGalvez, 2016).

\section{Tutoría entre iguales}

Dicha tutoría en la educación universitaria de Uruguay ha obtenido un impacto positivo, permitiendo por medio del trabajo colaborativo la creación de un vínculo diferente, a diferencia de las metodologías utilizadas habitualmente, esta ayuda al desarrollo de habilidades interpersonales y potencializa el aprendizaje en estudiantes tutores (Santiviago, Martínez, \& Nicaretta, 2015). En el espacio europeo de educación superior, es adaptable a cualquier contexto educativo, pero es necesaria una buena implementación del docente a cargo, su función como tutor debe ir integrada con los alumnos. La tutoría entre iguales no puede reemplazar la labor del docente, debe ser un medio de apoyo o complemento en la educación (Álvarez, 2015).

La tutoría entre iguales contribuye a mejorar la comprensión lectora y la fluidez, la cual es aplicada en la educación primaria en España, donde los resultados positivos son evidentes en el proceso de aprendizaje, esto debido a la superación de dificultades y la buena aceptación por los docentes a cargo, lo cual genera un ambiente propicio para su aplicación (Valdebenito \& Duran, 2013). En el programa "Leemos en parejas" se evidencia una evolución positiva, donde el intercambio informativo ha influido directamente en la mejora de la fluidez y comprensión lectora, cuya comunicación se evidencia en momentos claves como lo es, el antes y después de cada lectura. Lo anterior evidencia el potencial de la tutoría entre iguales para fortalecer la competencia lectora (Flores Coll \& Duran Gisbert, 2016).

\section{Tutoría en el contexto educativo}


Los diferentes modelos de aprendizaje implementados en contextos educativos Iberoamericanos de tutoría universitaria, muestran un apoyo desigual en la última década. El protagonismo en las instituciones se debe a una acción personalizada en el alumno, para poder orientarlo en su vida académica, profesional y personal. Se utilizan diferentes tipos de tutoría universitaria, tales como: Tutoría académica, personal, título o carrera, servicio, práctica e investigación, el gran porcentaje donde no se refleja la tutoría establece un desafío en la búsqueda de mejorar la calidad en la educación de América Latina (Lobato Fraile, \& Guerra Bilbao, 2016).

Las experiencias de alumnos en cursos iniciales de la Universidad de Cantabria, resalta la importancia de una dimensión tutorial personal, encontrando la necesidad de estos espacios en la institución, debido a los problemas evidenciados en los estudiantes nuevos, donde se ven enfrentados a un ambiente distinto, en el cual deben ser autónomos en su vida académica, esto es un proceso largo en distintos tipos de estudiantes, dificultando su adecuación. Debe existir un apoyo de las instituciones, lo cual permita generar espacios en el aula de clase para desarrollar la autonomía en cada uno de los estudiantes, reforzando la tutoría en la construcción de escenario de reflexión respecto a los cambios evidenciados al ingresar a la Universidad (Haya Salmón, Calvo Salvador, \& Rodríguez-Hoyos, 2013).

Se aplica la tutoría en el programa universitario "velero", el cual ofrece una serie de actividades académicas para brindar a los nuevos estudiantes una adaptación y un desarrollo integral en su nueva etapa universitaria. Las buenas valoraciones obtenidas en los estudiantes evidencian la importancia de un programa tutorial en la Universidad, el cual ofrezca un apoyo continuo para resolver las dudas o necesidades presentadas. El acompañamiento del docente es indispensable en el alumno para su desarrollo académico actual y profesional en un futuro (Pérez, 2013).

En un modelo de tutoría académica especial para estudiantes atletas de alto nivel, enfoca las dificultades de los atletas para integrar sus prácticas con el estudio académico, a veces por cuestiones físicas o por tiempo, lo cual hace necesario el uso de la tutoría en este tipo de comunidades estudiantiles, ofreciendo un 
acompañamiento continuo y ayudando así a superar dificultades e integrar sus formaciones académicas en búsqueda de superar posibles abandonos (Moreno \& Sallán, 2016)

\section{Enseñanza}

La enseñanza debe tener un conjunto de factores para su eficacia, donde se destaque la creación de un ambiente adecuado entre docente y alumno, una buena gestión del tiempo dedicado al proceso de enseñanza y el uso de una metodología adecuada, pero no solo la eficacia debe ser medida en lo realizado por el docente en el aula de clase, debe tenerse en cuenta aspectos de condiciones en donde se desarrolla el trabajo, las cuales son proporcionadas por las instituciones educativas, como lo es, contar con un buen equipo, una infraestructura adecuada y garantías laborales (Martínez-Garrido \& Murillo, 2016).

Enseñar con tecnologías digitales en el contexto universitario hace necesario la buena vinculación de docentes, donde debe existir una inclusión relacionada con los temas orientados. Los resultados de la implementación en la enseñanza a través de herramientas tecnológicas facilitan el aprendizaje, porque se pueden presentar cualquier tipo de temas con los medios tecnológicos utilizados actualmente (audios, videos, representaciones, entre otros), lo cual puede llegar a despertar el interés en los estudiantes (Marcelo, 2015). La utilización de las Tecnologías de la Información y la Comunicación (TIC) es vista como un recurso motivador e innovador en la educación, debido a la implementación de nuevas metodologías. El estudio de la aplicación en un centro de educación primaria detalla el escaso acceso a la tecnología, la poca dotación de profesores es determinante en los resultados. Los centros de educación secundaria muestran en cambio un mayor uso de las TIC, utilizada como un recurso con gran potencial en la creación de nuevos conocimientos, siendo también un facilitador en el rol docente, y en consecuencia generando resultados positivos (Garrido \& García, 2016).

La utilización de la APP en la educación potencializa su uso como herramienta de aprendizaje, donde es necesario la creación especial de un diseño pedagógico. Se plantea un modelo educativo divido en diferentes categorías para potencializar 
el aprendizaje por medio de las APP, brindando las siguientes: Buscar y filtrar, conocer y construir, aplicar y crear, analizar, colaborar, conectar y compartir, forman un ambiente personal y móvil de aprendizaje, facilitando de esta manera su utilización (Villalonga Gómez \& Marta-Lazo, 2015).

\section{Gamificación en la educación}

La gamificación es considerada una herramienta de motivación para aprender de una manera distinta cualquier tipo de tema, despertando la motivación y el interés del alumno por adquirir nuevos conocimientos, lo cual genera un reto en la construcción del aprendizaje por medio de juegos educativos (Oliva, 2017) (MeraPaz, 2016).

Estamos frente una generación digital con gran potencial de videojuegos en educación, lo cual exige replantear el concepto del aprendizaje actual (Simões, Redondo, \& Vilas, 2013). Tenemos como referente el juego de preguntas Kahoot, el cual se utiliza para fortalecer los conceptos en cualquier materia, creando una competencia sana dentro del grupo estudiantil y así obtener como resultado un interés del estudiante por aprender (Jiménez, Gámez, \& Gómez, 2016).

En el diseño de un Sistema Tutor Inteligente (STI) se utiliza la gamificación como parte de las estrategias de aprendizaje para conocer cuál es la respuesta de los estudiantes en la utilización del sistema tutor, el modulo se denomina "controlador de gamificación", simula un juego de puntos, encargado de la representación de bonos extras, lo cuales se identifican con estrellas representadas por una imagen, el cálculo de los puntos extras depende el tiempo de respuesta y esto genera una emoción la cual es detectada por el tutor a cargo (Zatarin-Cabada, Barrón-Estrada, \& García-Lizárraga, 2016).

\section{TIC's en la enseñanza}

La disponibilidad de las Tecnología de la Información y la Comunicación (TIC) en escuelas latinoamericanas inciden directamente en los resultados académicos. Cuando el uso de una computadora en el aula de clase es continuo se mejora el rendimiento académico, en caso contrario, genera una limitante en la construcción 
de nuevos conocimientos. Las escuelas Latinoamérica se enfrentan al desafío de aumentar la utilización de equipos tecnológicos en docentes y estudiantes (Román \& Murillo, 2014).

Los aspectos no tenidos en cuenta cuando se aplican nuevas metodologías relacionadas a las TIC, es la poca preparación del docente en la utilización de las mismas, generando un cuestionamiento respecto a como en la actualidad se están preparando los nuevos docentes, pero esto también se debe a la falta de inversión general de recursos económicos, humanos y de tiempo (Vaillant, 2014). La poca preparación de docentes en la mayoría de los casos se debe al poco apoyo de las instituciones, son sometidos a horarios pesados y no facilitan el tiempo necesario para poder prepararse en estos campos, su preparación debe ser continua a lo largo de su vida laboral en el campo académico (López \& Chávez, 2013).

La aplicación de la tecnología en educación para niños con Trastorno del Espectro Autista (TEA), debe generar un cambio de la organización escolar y de aula, existen una gran cantidad de herramientas para su aplicación, pero su uso se define específicamente para algunas características y el éxito depende de la integración de todos los actores relacionados con el aprendizaje. Se enfoca herramientas las cuales ayudan a mejorar y desarrollar las habilidades de los niños, donde no se enfoque la tecnología a utilizar, si no la persona, la cual hará uso de ella (Martínez, Pagán, García, \& Máiquez, 2016).

\section{Aprendizaje colaborativo}

Es una estrategia de enseñanza, la cual busca promover entornos colaborativos en la regulación individual y grupal de los estudiantes, teniendo en cuenta los componentes tanto motivacionales como afectivos relacionados en el trabajo colaborativo, lo cual permite un apoyo y una adaptabilidad con los estudiantes heterogéneos (Onrubia, Rochera, \& Angel, 2015).

La formación de los docentes en escenarios donde se aplica al Aprendizaje Colaborativo Apoyado por Computador (CSCL, por las siglas en inglés de Computer Supported Collaborative Learning), es fundamental orientar el aprendizaje colaborativo en las aulas de clases haciendo uso de las TIC, para ello es necesario 
realizar planes formativos, los cuales permitan ampliar los beneficios en el uso de herramientas tecnológicas, logrando obtener docentes apropiados en el tema y generadores de estrategias para situaciones presentadas en la implementación del trabajo colaborativo, teniendo en cuenta aspectos en donde se apoye directamente el proceso de aprendizaje (Avello-Martínez \& Marín, 2016).

Las experiencias del uso del trabajo colaborativo en un entorno virtual son acogidas de una manera positiva por los estudiantes, siendo un instrumento poderoso para el aprendizaje. En cada escenario del CSCL se deben evidenciar cambios en la implementación, los cuales han seguido una línea tradicional. Además, fortalecer los roles de cada uno de los participantes, partiendo de roles activos y centrados en fortalecer el aprendizaje. Es fundamental en la aplicación del CSCL estudiar a profundidad en el contexto el impacto social del trabajo colaborativo (Berns, Palomo-Duarte, Isla-Montes, Dodero, \& Delatorre, 2017).

El aprendizaje colaborativo por medio de blogs de una universidad muestra una gran herramienta potencial para diferentes usos dentro del aula estudiantil, ocasionando la descentralización de la función del docente, pero favoreciendo enormemente las habilidades personales en los estudiantes (Álvarez \& Bassa, 2013).

El trabajo colaborativo a través de seminarios potencializa el aprendizaje de nuevos conocimientos, ayuda de igual manera al desarrollo de nuevas habilidades, para la utilización de estos medios, es necesario crear un ambiente de aprendizaje autónomo, en donde se incluya la aplicación de lecturas individuales, grupales, fomentar el debate y la reflexión colaborativa (Vargas, Rojas, \& Castillo, 2019) (Borrasca, 2014).

\section{Entornos virtuales para la enseñanza}

Los principios constructivistas tenidos en cuenta en los entornos virtuales para la enseñanza deben relacionar las características individuales de cada estudiante en el aprendizaje y la adecuación al modelo educativo, debe existir una socialización entre los participantes. La autonomía es necesaria en todo proceso de aprendizaje, como también aspectos como la creatividad y el juego (García Aretio, 2016). En la 
educación secundaria el uso de las TIC es tomado como un tema de moda, lo cual debe cuestionarse y darle importancia como algo necesario para mejorar la calidad en la educación, porque ofrece una mejor manera de enseñar cualquier tema, activando el interés por aprender. Se hace fundamental su uso ante lo difícil de crear innovación en contextos actuales de educación (Chiappe, Mesa, \& Alvarez, 2013).

Las TIC en la educación a distancia tiene un gran acogimiento de los estudiantes por la metodología utilizada en la Universidad Nacional de Educación a Distancia (UNED), se toman aspectos importantes como lo es la tutoría o clase tutorial, los medios audiovisuales, las unidades y guías didácticas, forman el modelo de enseñanza utilizado en la universidad, el cual es considerado uno de las mejores en la enseñanza universitaria a distancia (Yáñez \& Navarro Fernández, 2013).

La evidencia documentada a lo largo de los años de un grupo de docentes sobre los proyectos de innovación educativa, enfocado en la contribución de los blogs como entornos virtuales en el proceso del aprendizaje-enseñanza de educación superior. Los resultados evidenciaron la importancia del uso de los blogs dentro de los procesos del aula, convirtiéndose en una herramienta para problemas presenciales de los alumnos, sea porque no pueden asistir o porque no se le facilita participar en clase. El uso de los blogs aumenta significativamente su participación, también con un buen seguimiento del docente, la interacción con los estudiantes crece, además genera un historial de lo hecho por el estudiante durante el curso, facilitando la evaluación de cada uno (Alventosa, Valcárcel, \& Valencia-Peris, 2015).

\section{Agentes Tutores en la educación virtual}

La educación virtual es una herramienta útil para mejorar los procesos llevados a cabo normalmente en la educación presencial, logrando acoger prácticas adaptables a los requerimientos de los cursos en diferentes plataformas virtuales (Durán, Estay-Niculcar, \& Álvarez, 2015). La tutoría inclusiva virtual en la educación a distancia ayuda directamente en el desarrollo cognitivo de los alumnos, pero es el tutor virtual quién beneficia la creación de nuevos ambientes colaborativos, es importante la generación de un impacto positivo en el estudiante, lo cual pueda reflejarse en el buen desarrollo cognitivo y genere un fortalecimiento de las 
relaciones interpersonales (Hernández-Sánchez \& Ortega, 2015). La educación virtual aplicada en la universidad establece una serie de retos para los participantes, se debe establecer el escenario virtual semejante a la universidad, para ello se hace necesario tener una buena interacción entre alumnos y maestros. Los temas académicos en entornos virtuales deben tener un alto grado de especificación, lo cual facilite la comprensión de tareas, por otra parte, los alumnos deben tener una gran autonomía en estos escenarios (Esteban \& Fuentes, 2015).

La tutoría virtual en la Universidad Nacional Abierta y a Distancia (UNAD) evidencio un buen comportamiento de los tutores en el aula virtual, lo cual ocasiona motivación para la realización de cursos por parte de estudiantes, porque se encuentran con una plataforma donde pueden solucionar sus problemas o necesidades del curso, siendo efectivo al responder las dudas, orientando a los usuarios cuando necesiten acudir a otras instancias. La utilización de la tutoría virtual se muestra como una herramienta facilitadora en ambientes de educación virtual, ayudando a formar estudiantes autónomos y responsables con sus deberes académicos (Estrada, 2015).

\section{E-Learning}

La tutoría en cursos de E-Learning debe replantear los roles establecidos para la educación virtual desde la perspectiva tutorial, teniendo por una parte las percepciones de los cursos en el alumnado y aclarando las estructuras de tutores en los cursos (Jiménez, Rodríguez, \& Hurtado, 2017). Las estrategias del aprendizaje emergente en el E-Learning enfatizan el estudio en la conformación de algunos factores de sus principales elementos, como lo son: factores de autorregulación, psicológicos, gestión y uso de la tecnología, estos factores determinan la garantía del éxito en el proceso de aprendizaje (Moreno, 2017).

La evolución el E-Learning a lo largo de los años relaciona un cambio en el concepto de diferentes autores, pero coinciden en tres avances principalmente: La enmarcada por la LMS, la web 2.0 y la separada de lo realizado por LMS avanzando hacia ecosistemas tecnológicos de aprendizaje. Es una herramienta considerada en general como ayuda en el proceso de aprendizaje-enseñanza, pero con un gran reto 
en la aplicación para instituciones donde no se evidencia un uso continuo de medios tecnológicos (García \& Seoane, 2015; Ortiz, Rojas, \& Cano, 2019).

La interfaz de un sistema E-Learning toma importancia en sus usuarios, a partir de ello pueden llegar a sentirse satisfechos y reflejarse en su continuo uso. El estudio de los indicadores de evaluación de calidad relacionó aspectos de interfaz como lo son: Lo atractivo en su usabilidad, el placer y el confort. Al tener un interfaz la cual cumpla con todos los requerimientos de sus usuarios, podrá ser un aspecto de ayuda para evitar los abandonos presentados en los cursos y aumentar el interés para su utilización (Aballay, Aciar, González, \& Collazos, 2016).

\section{Tutoría por medio de las TICs}

La eficacia de un programa el cual utiliza las TIC se logró evidenciar cuando es aplicada en una población de estudiantes de primaria, donde se enfocó la investigación en otros contextos, y se incluyeron otras etapas educativas en la enseñanza para resaltar la importancia del tutor y su relación con el estudiante (Luque \& Vallejo, 2017; Guayara, Millán, \& Gómez, 2019). La importancia de la tutoría para fortalecer la resiliencia presentada en estudiantes de educación superior, en la carrera universitaria de Ingeniería Ambiental de UPIBI, se realizó una encuesta para tener información acerca de los pasatiempos y la forma de realizar estudios en la comunidad universitaria, como también su interés al terminar la carrera, teniendo los resultados se analizó el motivo por el cual los estudiantes necesitan un acompañamiento tutorial. Se manifestó la poca interacción personalizada por parte del docente, relacionando el mal rendimiento académico de las materias donde no existe un acompañamiento. Lo anterior, podría ser uno de los motivos por el cual los alumnos no se sienten motivados para aprender, ocasionando abandonos en el curso (González, Monroy, \& Allende, 2017).

La labor del tutor con un alumno virtual con discapacidad, es vital para el éxito del programa brindar un acompañamiento adecuado, teniendo en cuenta aspectos como la preparación académica profesional en el área y la educación a las necesidades del alumnado, para con estas ayudar a superar los límites de las personas en situaciones especiales, las cuales solo buscan superarse y prepararse 
profesional y personalmente (Martínez, 2014). Es importante definir las características de los tutores en línea, entre los aspectos se destacan el ser agentes facilitadores dentro del entorno de aprendizaje, crear un ambiente idóneo es vital para lograr en los estudiantes el aprendizaje por medio de herramientas en línea y obtener al final una formación de calidad (García \& Domínguez, 2013).

\section{Sistemas tutores inteligentes en el ámbito de la educación}

Los sistemas tutores inteligentes en la educación, mejora los procesos en el ámbito estudiantil, ofreciendo una manera ágil e interactiva para la trasmisión de información en diferentes tipos de alumnos. Debido a los bajos resultados en matemática básica en pruebas ICFES se crea un Sistema Tutor Inteligente (STI) para apoyar el aprendizaje de matemáticas, ayudando a superar las debilidades del alumnado con diferentes estrategias aplicadas en el sistema (Hamidullah \& Samiullah, 2015). Los sistemas tutores inteligentes son considerados como una herramienta donde se puede lograr el proceso de aprendizaje-enseñanza, con un diseño adaptable según los tipos de estudiantes, con ello generar una adecuada metodología, lo cual permita alcanzar la adquisición de nuevos conocimientos (Hurtatiz, Rengifo, \& Rojas, 2015). La aplicación de un tutor inteligente reconoce y maneja las emociones presentadas en el aprendizaje, por medio de una red neuronal artificial, permitiendo extraer información acerca del aprendizaje obtenido y generar una retroalimentación la cual ayude en la calidad cuando se enseña un nuevo tema (Petrovica, Anohina-Naumeca, \& Ekenel, 2017)

\section{Discusión y conclusiones}

Los resultados analizados de las diferentes aplicaciones de los agentes tutores en la enseñanza, presenta investigaciones no sujetas a vacíos o futuros trabajos, impidiendo el desarrollo del tema a lo largo de los años.

La búsqueda realizada revela una gran cantidad de temas relevantes en las diferentes investigaciones, mostrando la tutoría como una estrategia clave en diferentes contextos académicos; educación a distancia, primaria, secundaria y universitaria, también sistemas especiales para cada alumno, siendo adaptable 
para estudiantes con alguna discapacidad. En la mayoría de trabajos el estudiante es el centro de todo el aprendizaje. Los resultados positivos en los centros educativos fortalecen la idea de ver la tutoría no como una metodología opcional, si no como la necesidad de resolver los problemas presentados en la educación, como son las diferentes desigualdades creadas por factores personales o sociales en los estudiantes, generando desinterés e impidiendo su normal desarrollo en la adquisición de nuevos conocimientos.

Algunos autores hablan de la aplicación de tutoría en el beneficio de la comprensión lectora (Valdebenito \& Duran, 2013; Flores \& Duran, 2016), pero no se estudia a profundidad los procesos establecidos en el intercambio informático, donde son estos procesos, los cuales permiten el éxito en su aplicabilidad, los artículos encontrados no abordan el tema, lo cual limita estudiar el comportamiento de los estudiantes en dichos procesos.

Las TIC se han utilizado en los últimos años para implementar sistemas tutores, es un medio muy efectivo e idóneo, en la aplicación debe existir mayor tiempo invertido por parte del docente y una autonomía mayor en el estudiante. En la tutoría virtual se presentan problemas como los posibles abandonos o la poca disposición de los docentes. No solo la utilización de nuevas tecnologías mejora el proceso de aprendizaje. La capacitación del docente es necesaria para una aplicación efectiva, además de las buenas prácticas dentro del aula, las condiciones ofrecidas por las instituciones educativas son factores importantes, porque inciden directamente en este proceso. En términos generales la tutoría aplicada en el aprendizaje es un trabajo conjunto entre el docente y la institución o centro educativo, lo cual garantiza las mejores condiciones para tutores y estudiantes.

\section{Referencias}

Aballay, L., Aciar, S., González, C., \& Collazos, C. (2016). Método de Medición de la Percepción de los usuarios sobre los sistemas E-Learning de los Centros e Instituciones Educativas. International Journal of Information Systems and Software Engineering for Big Companies, 3(1), 28-42

Álvarez, G., \& Bassa, L. (2013). TIC y aprendizaje colaborativo: el caso de un blog de aula para mejorar las habilidades de escritura de los estudiantes preuniversitarios. Universities and Knowledge Society Journal, 10(2), 5-19. https://doi.org/10.25267/hachetetepe.2017.v1.i14.9 
Agentes tutores para la enseñanza

Álvarez, M. (2015). La tutoría entre iguales en el marco del Espacio Europeo de Educación Superior: fortalezas y requisitos necesarios para su implantación. Revista de educación y derecho. Education and Law Review, (11), 1-18

Alventosa, P., Valcárcel, J., \& Valencia-Peris, A. (2015). Los blogs como entornos virtuales de enseñanza y aprendizaje en Educación Superior 1. Revista Complutense de Educación, 26, 15-31. https://doi.org/10.5209/rev rced.2015.v26.43791

Avello-Martínez, R., \& Marín, V. (2016). La necesaria formación de los docentes en aprendizaje colaborativo. Profesorado, 20(3), 687-713

Bacca-Cortés, Bladimir, Florián-Gaviria, Beatriz, García, Sergio, \& Rueda, Sebastian. (2017). Development of a platform for teaching basic programming using mobile robots. Revista Facultad de Ingeniería, 26(45), 6170. https://doi.org/10.19053/01211129.v26.n45.2017.6054

Berns, A., Palomo-Duarte, M., Isla-Montes, J., Dodero, J., \& Delatorre, P. (2017). Agenda colaborativa para el aprendizaje de idiomas: del papel al dispositivo móvil. Revista Iberoamericana de Educación a Distancia, 20(2), 119-139. https://doi.org/10.5944/ried.20.2.17713

Borrasca, B. (2014). El aprendizaje colaborativo en la universidad: referentes y práctica. Revista de Docencia Universitaria, 12(4), 281-302. https://doi.org/10.4995/redu.2014.5624

Chiappe, A., Mesa, N., \& Álvarez, C. (2013). Transformaciones en las Concepciones de los Docentes de Educación Secundaria acerca de la Web 2.0 y su uso en los procesos de enseñanza. Estudios pedagógicos (Valdivia), 39(2), 55-66. https://doi.org/10.4067/s0718-07052013000200004

Clares, P., Cusó, J., \& Juárez, M. (2014). Una (re) visión de la tutoría universitaria en los estudios de Grado. Revista de Docencia Universitaria, 12(1), 269-305. https://doi.org/10.4995/redu.2014.6425

Delgado, M., \& Ramos, S. (2017). La tutoría virtual en educación a distancia: tres casos de acompañamiento en ITESI. Pistas Educativas, 39(126), 10-18

Durán, R., Estay-Niculcar, C., \& Álvarez, H. (2015). Adopción de buenas prácticas en la educación virtual en la educación superior. Aula Abierta, 43(2), 77-86. https://doi.org/10.1016/.aula.2015.01.001

Esteban, F., \& Fuentes, J. (2015). La educación universitaria virtual: una reflexión en torno al cultivo de las virtudes. Teoría de la Educación. Revista Interuniversitaria, 27(1), 103-118. https://doi.org/10.14201/teoredu2015271103118

Estrada, M. (2015). Impacto que tiene en los estudiantes la atención de la tutoría virtual, impartida por parte de tutores diplomados en tutoría virtual de la UNAD. En Memorias Virtual Educa, Medellín, Colombia

Flores Coll, M., \& Duran Gisbert, D. (2016). Tutoría entre iguales y comprensión lectora, ¿un tándem eficaz? Los efectos de la tutoría entre iguales sobre la comprensión lectora. Universitas Psychologica, 15(2), 339352. https://doi.org/10.11144/javeriana.upsy15-2.teic

García, L. (2016). El juego y otros principios pedagógicos. Supervivencia en la educación a distancia y virtual. Revista Iberoamericana de Educación a Distancia, 19(2), 9-23. https://doi.org/10.5944/ried.19.2.16175

García, C., \& Domínguez, C. (2013). Tareas y competencias del tutor en línea. Profesorado, 17(2), 305-325

García-Peñalvo, F., \& Seoane Pardo, A. (2015). Una revisión actualizada del concepto de eLearning. Décimo Aniversario. Educación y Cultura en la Sociedad de la Información, 16(1), 119-144. https://doi.org/10.14201/eks2015161119144

Garrido, J., \& García, M. (2016). Las TIC en centros de Educación Primaria y Secundaria de Andalucía. Un estudio de casos a partir de buenas prácticas. Digital Education Review, 29, 134-165 
Gómez, E. (2013). La tutoría en la universidad: una experiencia innovadora en el Grado de Educación Primaria. Revista de Docencia Universitaria, 11(2), 243-261. https://doi.org/10.4995/redu.2013.5575

González, E., Monroy, A., \& Allende, L. (2017). El Acompañamiento Tutorial Para El Fortalecimiento de la Resiliencia En Estudiantes a Nivel Superior. Revista Electrónica sobre Tecnología, Educación y Sociedad, 4(7), 1-14

Guayara, C., Millan, E. E., \& Gómez, C. (2019). Diseño de un curso virtual de alfabetización digital para docentes de la Universidad de la Amazonia. Revista Cientifica, 1(34), 34-48. https://doi.org/10.14483/23448350.13314

Hamidullah, S., \& Samiullah, P. (2015). Intelligent tutoring system: Approaches, research and e-learning solution. En 8th International Workshop on Computational Intelligence and Applications, 53-58

Haya Salmón, I., Calvo Salvador, A., \& Rodríguez-Hoyos, C. (2013). La dimensión personal de la tutoría universitaria. Una investigación cualitativa en la Universidad de Cantabria (España). Revista Española de Orientación y Psicopedagogía, 24(3), 98-113. https://doi.org/10.5944/reop.vol.24.num.3.2013.11247

Hernández-Sánchez, A., \& Ortega, J. (2015). Aprendizaje electrónico afectivo: un modelo innovador para desarrollar una acción tutorial virtual de naturaleza inclusiva. Formación universitaria, 8(2), 19-26. https://doi.org/10.4067/s0718-50062015000200004

Hurtatiz, Y., Rengifo, Y., \& Rojas, E. (2015). Sistemas Tutores Inteligentes como apoyo en el proceso de aprendizaje. Redes de Ingeniería, 6(1), 25-44. https://doi.org/10.14483/udistrital.jour.redes.2015.1.a02

Jiménez, A., Gámez, J., \& Gómez, J. (2016). Una propuesta para el refuerzo de conceptos matemáticos a través de Kahoot. Revista del CIDUI, (3), 1-9

Jiménez, M., Rodríguez, E., \& Hurtado, J. (2017). Funciones de la tutoría en e-learning: estudio mixto de los roles del tutor online. Revista de Investigación Educativa, 35(2), 409-426. https://doi.org/10.6018/rie.35.2.273271

Lobato Fraile, C., \& Guerra Bilbao, N. (2016). La tutoría en la educación superior en Iberoamérica: Avances y desafíos. Educar, 52(2), 379-398

López de la Madrid, M., \& Chávez Espinoza, J. (2013). La formación de profesores universitarios en la aplicación de las TIC. Sinéctica, (41), 2-18

Luque, E., \& Vallejo, A. (2017). Eficacia de un programa de intervención basado en el uso de las TIC en la tutoría. Revista de Investigación Educativa, 35(1), 215-233. https://doi.org/10.6018/rie.35.1.248831

Marcelo, C. (2015). Enseñar con tecnologías digitales en la Universidad. Comunicar, 45(23), 117-124. https://doi.org/10.3916/c45-2015-12

Martínez, J., Pagán, F., García, S., \& Márquez, M. (2016). Las tecnologías de la información y comunicación (TIC) en el proceso de enseñanza y aprendizaje del alumnado con trastorno del espectro autista (TEA). Revista Fuentes, 14, 193-208

Martínez, L. (2014). El acompañamiento del alumno virtual. Revista Mexicana de Bachillerato a Distancia, 6(11), 69-73. https://doi.org/10.22201/cuaed.20074751e.2014.11.65010

Martínez, P., Pérez, J., \& Martínez, M. (2016). Las TIC y el entorno virtual para la tutoría universitaria. Educación XXI, 19(1), 287-310. https://doi.org/10.5944/educxx1.13942

Martínez-Garrido, C., \& Murillo, F. (2016). Investigación iberoamericana sobre enseñanza eficaz. Revista mexicana de investigación educativa, 21(69), 471-499 
Mera-Paz, J. A. (2016). Gamificación una estrategia de fortalecimiento en el aprendizaje de la ingeniería de sistemas, experiencia significativa en la Universidad Cooperativa de Colombia sede Popayán. Revista Científica, 3(26), 3-11. https://doi.org/10.14483/23448350.11085

Millán-Rojas, Edwin Eduardo, Gallego-Torres, Adriana Patricia, \& Chico-Vargas, Diana Carolina. (2016). Simulación de una red Grid con máquinas virtuales para crear un entorno de aprendizaje de la computación de alto desempeño. Revista Facultad de Ingeniería, 25(41), 85-92

Moreno, J., \& Sallán, J. (2016). Orientación y tutoría durante los estudios universitarios: el plan de acción tutorial. Revista Fuentes, 14, 172-192

Moreno, Y. (2017). Rol del Tutor en el Contexto del Aprendizaje Virtual. Revista Scientific, 2(6), 270-285. https://doi.org/10.29394/scientific.issn.2542-2987.2017.2.6.14.270-285

Narro Robles, J., \& Arredondo Galván, M. (2013). La tutoría: un proceso fundamental en la formación de los $\begin{array}{llll}\text { estudiantes } \quad \text { universitarios. } & \text { Perfiles }\end{array}$ https://doi.org/10.22201/iisue.24486167e.2013.141.40528

Oliva, H. (2017). La gamificación como estrategia metodológica en el contexto educativo universitario. Realidad y Reflexión, 16(44), 29-47. https://doi.org/10.5377/ryr.v44i0.3563

Onrubia, J., Rochera, M. J., \& Angel, A. (2015). Promover la regulación individual y grupal del aprendizaje en entornos colaborativos: una experiencia en Educación Superior. Electronic journal of research in educational psychology, 13(1), 189-210. https://doi.org/10.14204/ejrep.35.14058

Ortiz, J. D. A., Rojas, E. E. M., \& Cano, C. A. G. (2019). Aplicación de los metaversos y la realidad virtual en la enseñanza. Entre Ciencia e Ingeniería, 13(25), 59-67. https://doi.org/10.31908/19098367.4015

Park, S., \& Kim, C. (2015). Boosting learning-by-teaching in virtual tutoring. Computers \& Education, 82, 129140. https://doi.org/10.1016/j.compedu.2014.11.006

Pérez, P. (2013). La tutoría y la orientación universitaria en la nueva coyuntura de la enseñanza superior: el programa" Velero". Contextos Educativos. (8), 281-294. https://doi.org/10.18172/con.569

Petrovica, S., Anohina-Naumeca, A., \& Ekenel, H. (2017). Emotion Recognition in Affective Tutoring Systems: Collection of Ground-truth Data. Procedia Computer Science, 104, 437-444. https://doi.org/10.1016/i.procs.2017.01.157

Plaza-Galvez, L. F. (2016). Obstáculos presentes en Modelación Matemática. Caso Ecuaciones Diferenciales en la formación de Ingenieros. Revista Científica, 2(25), 176-187. https://doi.org/10.14483//udistrital.jour.RC.2016.25.a1

Ramírez-Montoya, Javier, Osuna-Vergara, Ignacio, Rojas-Mora, Jessica, \& Guerrero-Gómez, Stalyn. (2016). Remuestreo Bootstrap y Jackknife en confiabilidad: Caso Exponencial y Weibull. Revista Facultad de Ingeniería, 25(41), 55-62

Rodríguez, M. (2013). Las TIC en Tutoría. Revista de Claseshistoria, (6), 2

Román, M., \& Murillo, F. (2014). Disponibilidad y uso de TIC en escuelas latinoamericanas: incidencia en el rendimiento escolar. Educação e Pesquisa, 40(4), 869-895. https://doi.org/10.1590/s1517$\underline{97022014121528}$

Santiviago, C., Martínez, A., \& Nicaretta, F. (2015). Tutorías entre iguales de interfase: una práctica innovadora. Revista latinoamericana de educación inclusiva, 9(2), 59-71

Simões, J., Redondo, R., \& Vilas, A. (2013). A social gamification framework for a K-6 learning platform. Computers in Human Behavior, 29(2), 345-353. https://doi.org/10.1016/j.chb.2012.06.007

Vaillant, D. (2014). Formación de profesores en escenarios TIC. Revista e-Curriculum, 12(2), 1128-1142 
Valdebenito Zambrano, V., \& Duran Gisbert, D. (2013). La tutoría entre iguales como un potente recurso de aprendizaje entre alumnos: efectos, fluidez y comprensión lectora. Perspectiva Educacional, Formación de Profesores, 52(2), 154-176. https://doi.org/10.4151/07189729-vol.52-iss.2-art.141

Vallejo Giraldo, F. (2017). Acompañamiento tutorial en la Universidad de Antioquia. Revista Virtual Universidad Católica del Norte (52), 3-22

Vargas, Y. A. M., Rojas, E. E. M., \& Castillo, V. S. (2019). Application of augmented reality as a means of interdisciplinary learning. Scientia et technica, 24(3), 481-491

Villalonga Gómez, C., \& Marta-Lazo, C. (2015). Modelo de integración educomunicativa de 'apps' móviles para la enseñanza y aprendizaje. Pixel-Bit, (46), 137-153

Yáñez, E., \& Navarro Fernández, J. (2013). Evaluación del uso de tecnologías en la enseñanza universitaria a distancia de la UNED. Revista Iberoamericana de Educación a Distancia, 16(2), 73-106. https://doi.org/10.5944/ried.16.2.9907

Zatarain-Cabada, R., Barrón-Estrada, M., \& García-Lizárraga, J. (2016). Sistema tutor afectivo para el aprendizaje de las matemáticas usando técnicas de gamificación. Research in Computing Science, 111, 83-96. https://doi.org/10.13053/rcs-111-1-7 